\title{
Iterated stretching, extensional rheology and formation of beads-on-a-string structures in polymer solutions
}

\author{
Mónica S.N. Oliveira, Roger Yeh, Gareth H. McKinley* \\ Hatsopoulos Microfluids Laboratory, Department of Mechanical Engineering, Massachusetts Institute of Technology, Cambridge, MA 02139, USA
}

Received 28 June 2005; accepted 20 January 2006

\begin{abstract}
The transient extensional rheology and the dynamics of elastocapillary thinning in aqueous solutions of polyethylene oxide (PEO) are studied with high-speed digital video microscopy. At long times, the evolution of the thread radius deviates from self-similar exponential decay and competition between elastic, capillary and inertial forces leads to the formation of a periodic array of beads connected by axially uniform ligaments. This configuration is unstable and successive instabilities propagate from the necks connecting the beads and ligaments. This iterated process results in multiple generations of beads developing along the string in general agreement with predictions of Chang et al. [Phys. Fluids, 11 (1999) 1717] although the experiments yield a different recursion relation between the successive generations of beads. At long times, finite extensibility truncates the iterated instability, and slow axial translation of the bead arrays along the interconnecting threads leads to progressive coalescence before the ultimate rupture of the fluid column. Despite these dynamical complexities it is still possible to measure the steady growth in the transient extensional viscosity by monitoring the slow capillary-driven thinning in the cylindrical ligaments between beads.
\end{abstract}

(C) 2006 Elsevier B.V. All rights reserved.

Keywords: Extensional rheology; Beads on a string; Self-similarity; Iterative process

\section{Introduction}

It has been known for at least 40 years that the dynamics of capillary thinning and breakup of polymeric jets and threads is substantially different from the equivalent processes in Newtonian fluids $[1,2]$. The capillary necking induced by surface tension results in a strong uniaxial stretching flow in the thread and leads to large molecular elongation. The resulting viscoelastic stresses in the fluid inhibit the finite time singularity associated with breakup in a Newtonian fluid jet $[3,4]$. The large viscoelastic stresses arising from the stretching can also result in the formation of a characteristic morphology known as a beads-on-a-string structure in which spherical fluid droplets are interconnected by long thin fluid ligaments. Understanding the distribution of the droplets resulting from the dynamics of this process is important in numerous commercial applications including jet breakup [5], fertilizer spraying [6], high-speed atomization [7], forward roll-coating and other coating applications [8], electrospinning [9] and inkjet printing [10]. Additional details of many of these applications are provided in the monograph by Yarin

\footnotetext{
* Corresponding author. Tel.: +1 617258 0754; fax: +1 6172588559 .

E-mail address: gareth@mit.edu (G.H. McKinley).
}

[11]. Similar beads-on-a-string structures have also been documented recently during gravitationally driven stretching of fluid threads formed from wormlike micellar solutions [12].

The most complete investigation of the formation of beadson-a-string (BOAS) structures was performed by Goldin et al. [2]. They studied aqueous solutions of polyethylene oxide and polyacrylamide and used stroboscopic flash photography to document the evolution of high-speed laminar jets. In addition to observing the formation of thin interconnecting elastic ligaments of random lengths they documented the development of small secondary droplets and speculated that large increases in the extensional viscosity of the polymer solutions were important for stabilizing such structures. Schümmer and Tebel [13] recognized that the formation of a long thin filament undergoing uniaxial elongation under the action of capillarity could be used as the basis of a 'free-jet' extensional rheometer. By using a simple force balance for the filament and analyzing high-speed photographs, the evolution in the extensional viscosity of the filament could be evaluated. More recently Christanti and Walker $[14,15]$ used a periodically forced jet to study the role of viscoelasticity in controlling jet stability to different wavelength disturbances and the subsequent formation of primary drops. They also measured the distribution of sizes of the secondary 
drops which developed on the thin interconnecting ligaments between the primary drops. For the most elastic solutions a second generation of beads between the primary drops was clearly visible.

However, in all of these previous studies it proved difficult to observe the very long time dynamics of the drop formation and breakup process (such as the development of third generation droplets) because long times correspond to material elements of fixed Lagrangian identity being convected large distances downstream of the nozzle. Under such conditions the high-speed jet is typically susceptible to infinitesimal external perturbations. In the present experiments we use a liquid bridge arrangement in which the background jet velocity is zero; instead a rapid axial strain is initially imposed to generate a long slender fluid thread of the polymer solution and this thread subsequently undergoes capillary-driven drainage and breakup. The fixed endplates that confine the experimental sample ensure that convective processes are not important and the stationary Eulerian nature of the resulting filament enables us to monitor its evolution using both a laser micrometer and high-speed digital video. Furthermore, the small axial and lateral dimensions of the fluid thread ensure that gravitational effects do not perturb the dynamics of breakup.

The formation of a beads-on-a-string morphology is inherently a nonlinear dynamical process. Classical linear stability analysis shows that a viscoelastic fluid thread with zero initial polymeric stresses in the material is in fact more unstable than a Newtonian fluid of equivalent steady state shear viscosity. This is due to the temporal retardation of the viscoelastic stresses that develop in the fluid as the result of a perturbation of any specified wavelength [1,2]. However, the uniaxial extension in the neck region (in which the perturbation has maximum amplitude) results in exponential growth of the polymeric stresses in the thinning filament. These elastic stresses suppress further growth in the disturbance and both asymptotic analyses $[4,16]$ and numerical simulations $[17,18]$ show the formation of an axially uniform thread or ligament connecting two drops. In the spherical beads, the molecules are relaxed and surface tension dominates; whereas in the thin thread the molecules are highly stretched and viscoelastic stresses dominate.

In a very thorough investigation of the linear and nonlinear dynamics of the slender filament equations derived for a finitely extensible nonlinear elastic (FENE) dumbbell model, Chang et al. [19] predicted that at long times an additional phenomenon, coined 'iterated stretching', should develop for low viscosity elastic fluids in which elasticity, capillarity and inertia are all important. In this stage of the dynamics, the neck region connecting the cylindrical thread to the spherical bead was shown to be unstable to perturbations which triggered a new instability and an "elastic recoil" close to the neck. This recoil leads to the formation of a smaller "secondary" spherical drop connected to the primary drop by a new thinner cylindrical thread. This new thread subsequently thins under the action of capillarity and the necks connecting the thread to the primary drop and new secondary drop may once again become unstable. This hierarchical process can repeat itself indefinitely, provided that the molecules have not reached full extension, leading to multiple generations of beads on strings. Fluid inertia is important in the develop- ment of this morphology because the disturbances in the neck region must grow sufficiently fast to exceed the rate of capillary thinning in the primary filament. As noted elsewhere [20], the elastocapillary thinning rate of the thread scales with $\lambda^{-1}$ (where $\lambda$ is the characteristic polymer relaxation time) and the growth rate of disturbances to the thread scale with the inverse of the Rayleigh time scale, $\left(\sigma / \rho R^{3}\right)^{1 / 2}$ (with $\sigma$ the surface tension and $\rho$ the density of a fluid thread of radius $R$ ). We thus require the natural or 'intrinsic' Deborah number $\lambda \sigma^{1 / 2} /\left(\rho R^{3}\right)^{1 / 2} \gtrsim 1$ to observe a repeated instability and the formation of multiple generations of beads-on-a-string.

Similar iterated instabilities have been predicted numerically and observed experimentally in viscous Newtonian fluid threads $[21,22]$. However, these iterated processes do not lead to the formation of a stable beads-on-a-string structure because each successive iteration leads to a thinner filament that is more unstable to perturbations. There are no viscoelastic stresses to stabilize the rapid growth of disturbances that develop in the thinnest necks and the thread rapidly ruptures.

Iterated capillary breakup processes in protoplasmic threads were also discussed very early on by D'Arcy Thompson [23; see pp. 65-66] and distinctive features that may be recognized as having the characteristics of a 'blobs'-on-a-string structure are described and sketched for the breakup of a non-Newtonian fluid (a cylinder of viscoelastic cellular cytoplasm) surrounded by an immiscible lower viscosity fluid. A similar two-fluid system with high viscosity contrast was also utilized by Chang et al. [19] to obtain some preliminary images of iterated stretching events.

In a recent letter [24] we documented the iterated stretching phenomenon experimentally for the first time using a viscoelastic fluid thread in air. A thin thread was formed between two cylindrical plates in a capillary breakup extensional rheometer (CABER) and the evolution into beads was followed using a digital video-microscope. The CABER device is typically used to measure the transient extensional viscosity of complex fluids [25]. In dilute polymer solutions the extensional viscosity is expected to be much larger than the steady shear viscosity and it will depend on the molecular weight of the polymer, the polymer concentration and chain flexibility [26-28]. It is very hard to quantitatively evaluate the extensional viscosity for such fluids using other techniques [29]; however, monitoring the slow capillary drainage and ultimate rupture of a necking fluid thread in a variety of dripping/jetting configurations can provide a suitable way of measuring this elusive material function $[3,27,28,30]$.

The analysis of the capillary thinning process - on which computation of an extensional viscosity is predicated - assumes that the fluid thread is a slender cylindrical filament. The development of an axially periodic structure along the filament may well thus be expected to compromise the efficacy of the instrument in measuring the extensional viscosity of the fluid. Indeed, if a laser micrometer alone is used to measure the decay in the filament diameter at the axial midplane, then periodic fluctuations in the signal can be detected in low viscosity elastic solutions [26,31] and these disturbances prevent a meaningful computation of the extensional viscosity. However, the analysis of Chang et al. [19], together with recent numerical simulations of Li and Fontelos [18], show that in the thin interconnecting ligaments 
between the beads an elasto-capillary balance still holds. In the present paper we analyze these regions using an imaging system that is capable of both high spatial resolution $( \pm 2 \mu \mathrm{m})$ and high temporal resolution $( \pm 0.001 \mathrm{~s})$, and use the results to evaluate the transient extensional stress growth in the fluid. In Section 2 we describe the rheology of the viscoelastic test fluid and additional details of the characterization technique. In Section 3 we present detailed observations of the evolution of the thread radius with time, and document the onset of instability at the junction between the bead and the interconnecting elastic ligaments, and the rapid formation of a hierarchical structure followed by a prolonged coalescence phase. Finally, we use these observations to evaluate the growth in the transient extensional viscosity and the approach to a steady state value corresponding to an apparent Trouton ratio in excess of $10^{4}$.

\section{Experimental methods and data analysis}

To observe iterated stretching and the development of a multigenerational beads-on-a-string structure, a number of key physical conditions must be realized [19]. The thinning of a polymer solution described by a nonlinear constitutive equation such as the FENE model is controlled by multiple physical parameters that can be combined to give four dimensionless parameters; a Deborah number, defined above as a ratio of the polymer relaxation time to the Rayleigh time scale for inertio-capillary breakup of a thread of initial radius $r_{0}$, $\mathrm{De}=\lambda / \sqrt{\rho r_{0}^{3} / \sigma}$; an Ohnesorge number characterizing the relative importance of viscous effects in the inertio-capillary breakup process of a fluid thread $\mathrm{Oh}=\eta_{0} / \sqrt{\rho \sigma r_{0}}$; a solvent viscosity ratio $S=\eta_{\mathrm{s}} /\left(\eta_{\mathrm{s}}+\eta_{\mathrm{p}}\right)=\eta_{\mathrm{s}} / \eta_{0}$ characterizing the individual contributions of the background solvent $\left(\eta_{\mathrm{s}}\right)$ and the polymer $\left(\eta_{\mathrm{p}}\right)$ to the total viscosity and, finally, a finite extensibility parameter $L$ that characterizes the ratio of the maximum length to the equilibrium length of the polymer molecules. This dimensionless parameter is a ratio of the contour length of the macromolecule to the radius of gyration in solution and scales with the square root of the molecular weight of the solute [32]: $L \sim M_{\mathrm{W}}^{1 / 2}$. In these definitions, $\rho$ is the density of the fluid, $\eta_{\mathrm{s}}$ the solvent viscosity and $\eta_{0}$ is the total zero-shear-rate viscosity. The Ohnesorge number provides a dimensionless measure of the relative importance of viscous and inertial effects in unforced jet breakup, and is inversely related to a local Reynolds number for the flow $R e \sim \mathrm{Oh}^{-2}=\rho V_{\text {cap }} r_{0} / \eta_{0}$ where $V_{\text {cap }}$ is a capillary velocity $V_{\text {cap }}=\sigma / \eta_{0}$.

All previous numerical predictions and experimental studies of capillary thinning and viscoelastic thread breakup can be represented in different regimes of this four-dimensional parameter space. In particular, Chang et al. [19] demonstrated that for iterated stretching to be observed one requires high Deborah numbers $D e \gg 1$, intermediate viscosity ratios $(0<S<1)$, finite fluid inertia $\mathrm{Oh} \sim O(1)$ - so that inertial effects lead to rapid growth of the capillary instability and recoil - plus very high finite extensibilities $L \gg 1$ so that the iterated nature of the instability and elastic recoil process is not truncated prematurely by the maximum length of the molecules.
Table 1

Viscometric and physical properties of the viscoelastic solutions used in the experiments

\begin{tabular}{lllll}
\hline$\eta_{\mathrm{P}}(\mathrm{mPas})$ & $\eta_{\mathrm{S}}(\mathrm{mPas})$ & $\eta_{0}(\mathrm{mPas})$ & $\sigma(\mathrm{N} / \mathrm{m})$ & $\rho\left(\mathrm{g} \mathrm{cm}^{-3}\right)$ \\
\hline 40.32 & 6.77 & 47.09 & 0.0623 & 0.925 \\
\hline
\end{tabular}

To obtain such values experimentally, we use a high molecular weight water-soluble flexible polymer, poly(ethylene oxide) or PEO, commonly used in drag reduction [33] and viscoelastic jet breakup studies [14,34]. The specific grade of polymer used (WSR-301) is commercially available (Union Carbide) and polydisperse, with a molecular weight $\bar{M}_{\mathrm{W}}$ that is difficult to characterize precisely because of aggregation [35] but is in excess of $3.8 \times 10^{6} \mathrm{~g} / \mathrm{mol}$. Using published correlations [36] this corresponds to an intrinsic viscosity of $[\eta]_{0} \approx 1.42 \times 10^{+3} \mathrm{~cm}^{3} / \mathrm{g}$ and an overlap concentration of $c^{*} \sim 1 /[\eta]=590 \mathrm{ppm}$. Direct measurements of intrinsic viscosities of different PEO samples over a range of molecular weights show that the solvent quality parameter is $v \approx 0.56$ [34]. The high molecular weight and flexibility of the PEO chains result in a high value of the FENE extensibility parameter; using published values for the characteristic ratio $C_{\infty}$ and the molecular bond lengths we obtain $L^{2} \approx 2.4 \times 10^{4}[34]$.

The polymer is dissolved at a concentration of $2000 \mathrm{ppm}$ in a mixture of ethylene glycol and water to give a semidilute viscoelastic polymer solution $\left(c / c^{*}=3.4\right)$ with the viscometric properties shown in Table 1 . The variation in the steady shear viscosity with shear rate measured with a cone-and-plate fixture $\left(\theta_{0}=0.0177 \mathrm{rad}\left(1^{\circ} 1^{\prime}\right)\right)$ is shown in Fig. 1(a). Rheological reproducibility and accuracy becomes difficult for low viscosity fluids at high shear rates. We therefore show in Fig. 1 the measured viscosity of a Newtonian calibration oil with viscosity $\mu=0.138 \mathrm{~Pa}$ s. The PEO solution has a zero-shear-rate viscosity of $0.047 \mathrm{Pas}$ and begins to shear thin gradually at shear rates of $\dot{\gamma} \sim 2 \mathrm{~s}^{-1}$. The measured viscosity decreases monotonically until a shear rate of $\dot{\gamma}=350 \mathrm{~s}^{-1}$. At this point a torsional flow instability leads to an increase in the apparent viscosity. The appropriate Reynolds number [37] at this point is $R e=$ $\rho(\Omega R)\left(R \theta_{0}\right) / \eta(\dot{\gamma})=8.3$ (where $\Omega$ is the rotational velocity of the fixture) and the relevant viscoelastic parameter for parameterizing the instability [38,39] is $W_{\mathrm{i}} \sqrt{\theta_{0}}=\lambda \dot{\gamma} \sqrt{\theta_{0}} \approx 11$. Both inertial and elastic effects would thus appear to be important in controlling the critical conditions for this instability [40]; however further exploration of this is beyond the scope of the present work. The first normal stress difference of this low viscosity elastic fluid is below the measurable resolution of the AR1000N rheometer. In Fig. 1(b) we show the linear viscoelastic properties of the $2000 \mathrm{ppm}$ PEO solution. The storage and loss moduli exhibit the expected Zimm-like frequency-dependence for dilute solutions of flexible polymers [41]; however, great care must be taken with these low viscosity elastic fluids in order to not exceed the linear viscoelastic limits of the material. Two sets of measurements are shown at oscillating stresses of magnitudes 0.05 and $0.005 \mathrm{~Pa}$, respectively. Good superposition is obtained across the frequency range. At angular frequencies above $10 \mathrm{rad} / \mathrm{s}$ inertial effects overwhelm the small elastic (in- 

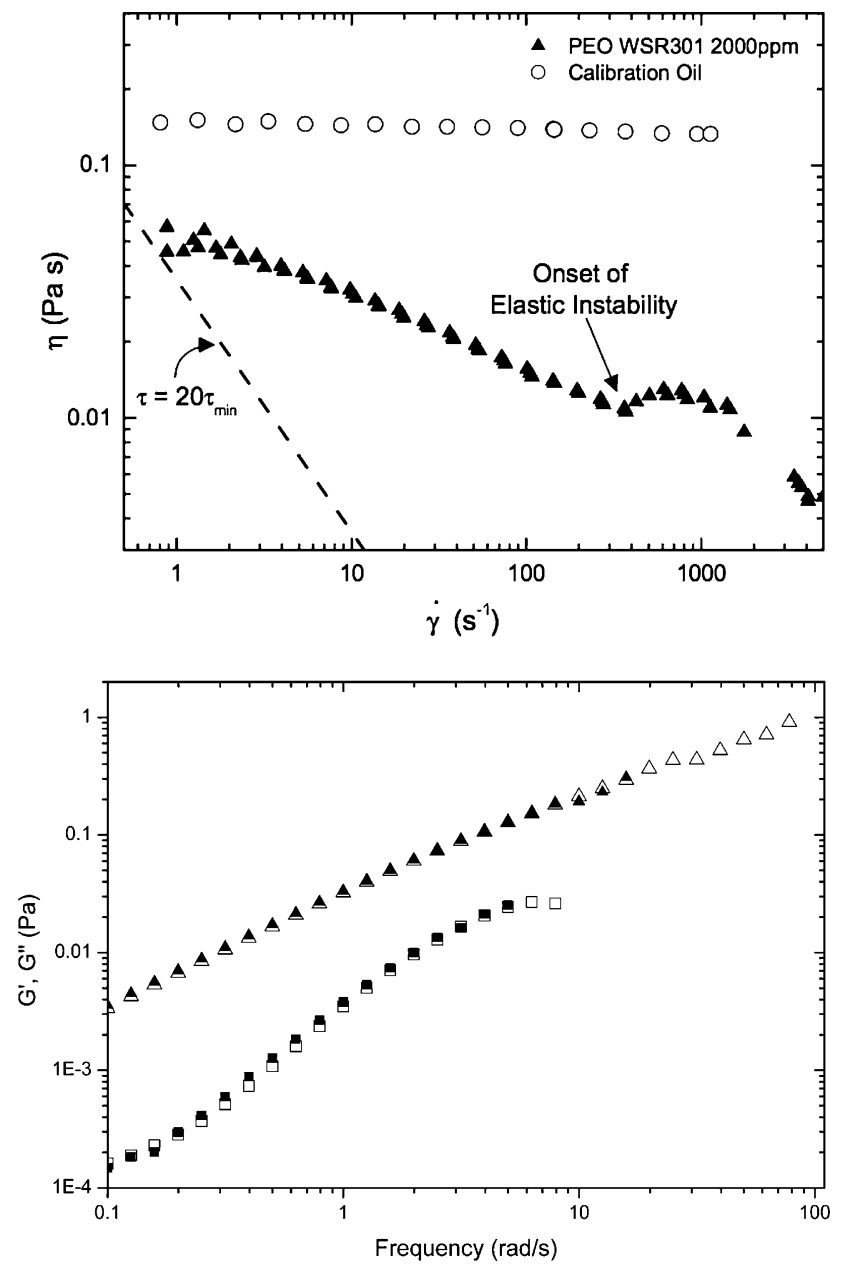

Fig. 1. Rheological properties of the 2000 ppm PEO (WSR-301) solution: (a) steady shear viscosity showing the shear-thinning region and the onset of a torsional viscoelastic instability at a critical rotation rate; (b) frequency-dependence of the storage modulus (squares) and loss modulus (triangles) at fixed stress amplitudes of $0.05 \mathrm{~Pa}$ (hollow symbols) and $0.005 \mathrm{~Pa}$ (filled symbols).

phase) contribution to the signal and the resulting data points are discarded.

A characteristic relaxation time may be estimated from the storage moduli by identifying the point at which a pronounced deviation from the terminal scaling $G^{\prime} \sim \omega^{2}$ is observed. This yields an estimate of $\omega^{*} \approx 5 \mathrm{rad} / \mathrm{s}$ and a time constant of order $\lambda \sim 1 / \omega^{*} \approx 0.2 \mathrm{~s}$; however, it is clear that a definitive and unambiguous determination is not readily possible from the linear viscoelastic data available. We therefore use capillary thinning experiments in order to determine the fluid time scale relevant for extensional flow. The measurements (described in detail below) give a fluid relaxation time of $\lambda \approx 0.23 \pm 0.02 \mathrm{~s}$ for a concentration of $2000 \mathrm{ppm}$. We have performed additional rheological experiments with different polymer concentrations and find that the relaxation time and the polymeric contribution to the viscosity both exhibit a dependence on concentration that scales approximately as $\sqrt{c}$ in agreement with other measurements in dilute and semidilute aqueous solutions [42,43]. As the concentration is varied, the dimensionless parameters $S$, Oh and De - which are relevant to formation of beads-on-a-string structure - also vary. At higher polymer concentrations, the increase in the fluid viscosity (and the correspondingly larger value of $\mathrm{Oh}$ ) results in an axially uniform and long-lived filament of the shape observed in capillary-thinning experiments with highly elastic Boger fluids $[28,44]$ and no bead formation. Conversely, at lower concentrations and/or lower molecular weights, the very low viscosity of the solution results in an initial phase of rapid inertio-capillary pinching, followed by formation of a single large 'primary droplet' that is centrally located between the two circular end-plates. This structure was documented recently using high-speed video-imaging by Rodd et al. [31] and the drainage of this structure under influence of gravity precludes accurate measurement of the transient elongational viscosity [26]. Once again no periodic beads-on-a-string structure is observed. Capillary-thinning experiments show that the most pronounced structures develop for the $2000 \mathrm{ppm}$ solution and we thus focus our attention henceforth on this fluid.

Using the measured relaxation time of $0.23 \mathrm{~s}$, we find that the Deborah numbers in fluid threads of initial diameter $2 r_{0} \approx 1.2 \mathrm{~mm}$ are $\mathrm{De} \geq 127$. Eggers [45] notes that inertial, viscous and capillary effects will all become important in a necking fluid thread (i.e. such that $\mathrm{Oh} \sim 1$ ) on length scales $\ell \sim \eta_{0}^{2} / \rho \sigma$. For the fluid properties given in Table 1 this corresponds to $\ell \approx 39 \mu \mathrm{m}$. For low viscosity Newtonian fluids the ensuing iterated necking events will evolve on time scales $t_{\text {Rayleigh }}=\sqrt{\rho \ell^{3} / \sigma} \approx 30 \mu \mathrm{s}$. Viscoelastic effects are expected to slow down the filament dynamics; however, it is clear that the necking and evolution of the beads-on-a-string microstructure will evolve rapidly in time and on fine length scales. We thus use a high-speed digital CMOS video camera (Phantom 5) operating at frame rates of 1600-1800 fps in conjunction with a high-resolution video-microscope lens system (Infinity K2 with an objective lens giving a spatial resolution of $\sim 2.3 \mu \mathrm{m}$ per pixel) to resolve the late stage dynamics.

\subsection{Analysis}

Analyses of the necking phase of the dynamics of elastocapillary thinning commonly make use of simplified 'zerodimensional' analyses in which axial variations along the filament are neglected entirely and the thread is considered to be infinitely long with a spatially uniform but time-varying radius $R(t)$. The resulting force balance can be written in the form [46]:

$3 \eta_{\mathrm{s}}\left\{\frac{2}{R(t)} \frac{\mathrm{d} R}{\mathrm{~d} t}\right\}=\frac{\sigma}{R(t)}-\Delta \tau_{\mathrm{p}}(t)$

in which the three terms represent, respectively, the stress contributions of a viscous solvent undergoing uniaxial elongation, the capillary pressure and the elastic stress difference $\left(\Delta \tau_{\mathrm{p}}=\tau_{\mathrm{p}, \mathrm{zz}}-\tau_{\mathrm{p}, \mathrm{rr}}\right)$ in the thread.

Combining this force balance with a quasilinear constitutive equation such as the Oldroyd-B model [47] shows that there is an exponential growth of the elastic tensile stresses, which is accompanied by an exponential decay in the filament radius (and concomitant increase in the capillary pressure within the jet). The contribution of the viscous solvent becomes negligi- 
bly small and the resulting elasto-capillary balance results in the following predicted rate of thinning in the filament radius [48]:

$$
R(t) / R_{0}=\left(\eta_{\mathrm{p}} R_{0} / \lambda \sigma\right)^{1 / 3} \exp (-t / 3 \lambda)
$$

where $R_{0}$ is the initial radius of the filament, $\eta_{\mathrm{p}}$ the polymer viscosity, $\lambda$ the relaxation time and $\sigma$ is the surface tension. Direct measurement of this rate of decay using a laser micrometer or a digital video camera thus enables construction of a capillary-thinning extensional rheometer which provides quantitative determination of the characteristic relaxation time of the fluid $[25,26,28,44]$.

Of course, such a zero-dimensional analysis cannot capture axial structures such as the growth of a beads-on-a-string morphology. Recent theoretical analyses and high resolution numerical simulations of one-dimensional slender filament models derived from the governing conservation equations have shown that the full profile also evolves in a self-similar manner; the precise dynamics depend on the relative magnitudes of the inertial, viscous, elastic and capillary terms in the governing equation (see Eggers [45], Renardy [49] and McKinley [20] for detailed reviews).

Ultimately this exponential thinning of the viscoelastic fluid thread is truncated by the maximum elongation of the macromolecules in solution. This finite extensibility truncates the exponential stress growth and the thread is then expected to thin linearly in time towards a breakup event with a general form $R(t) \sim\left(\sigma / \eta_{\mathrm{E}}\right)\left(t_{\mathrm{c}}-t\right)$ where $\eta_{\mathrm{E}}$ is the steady extensional viscosity. With this form of evolution in the radial profile, the tensile stress and the strain rate in Eq. (1) both diverge as $\dot{\varepsilon}_{\text {mid }} \sim\left(t_{\mathrm{c}}-t\right)^{-1}$. The precise value of the numerical front factor in this expression depends on the specific nonlinear constitutive model selected and the resulting value of the steady elongational viscosity at large strains and strain rates [50-52].

\section{Results}

In Fig. 2 we show a representative set of measurements of the global thinning dynamics in a Capillary Breakup Extensional Rheometer (CABER-1, Cambridge Polymer Group). Initially, the $6 \mathrm{~mm}$-diameter plates are separated by a gap $h_{i}=3 \mathrm{~mm}$ as seen in Fig. 2(a1) corresponding to an aspect ratio $\Lambda_{i}=h_{i} / R_{\mathrm{p}}=1$. The liquid bridge confined between the plates is stretched as the top plate moves linearly $(-50 \mathrm{~ms} \leq t \leq 0)$ to a specified distance $h_{0}=9.7 \mathrm{~mm}$ (Fig. 2(a2)). The length of the fluid thread now exceeds the Plateau stability limit and the system selects its own necking dynamics so that the viscous, elastic, capillary (and gravitational) forces balance each other. A laser micrometer (Omron Z4LA), measures the evolution of the midpoint filament diameter, $D_{\text {mid }}(t)=2 R_{\text {mid }}(t)$ as the thread thins under the action of capillarity and eventually breaks at a time denoted $t_{\mathrm{f}}$. A number of different regimes can be discerned in the data shown in Fig. 2(b). Shortly after the top plate comes to a halt (for times $0 \leq t \leq 45 \mathrm{~ms}$ ), inertio-capillary oscillations of the hemispherical fluid droplets attached to the end plates occur. These oscillations (with period $T \approx(\pi / \sqrt{2}) t_{R}$ corresponding to damped oscillations of a viscous liquid globe [53]) decay through the action of fluid viscosity and forthwith these regions act as quasi-static fluid reservoirs into which fluid from the necking thread can drain. The damped oscillations are followed by the rapid development of a central axially-uniform connecting filament of initial diameter $2 r_{0} \approx 1.1 \mathrm{~mm}$ which drains very slowly. On these intermediate time and length scales, inertial, viscous and gravitational effects can be neglected and a balance between surface tension and elasticity governs the filament drainage [48]. In this regime, the local extensional rate in the filament is constant with magnitude $\dot{\varepsilon}_{\text {mid }}=2 /(3 \lambda)$ and its diameter decays exponentially with time according to Eq. (2). The characteristic relaxation time is extracted by fitting the data to this expression, yielding $\lambda=228.5 \pm 1.8 \mathrm{~ms}$ (solid line in Fig. 2(b)).
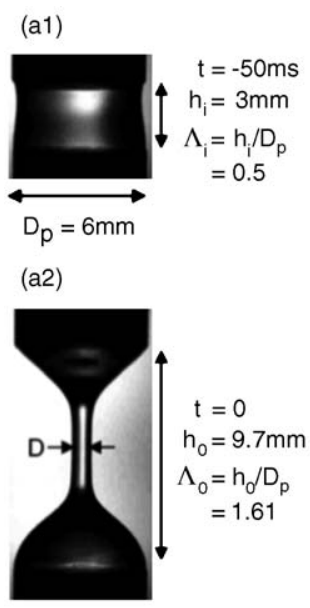

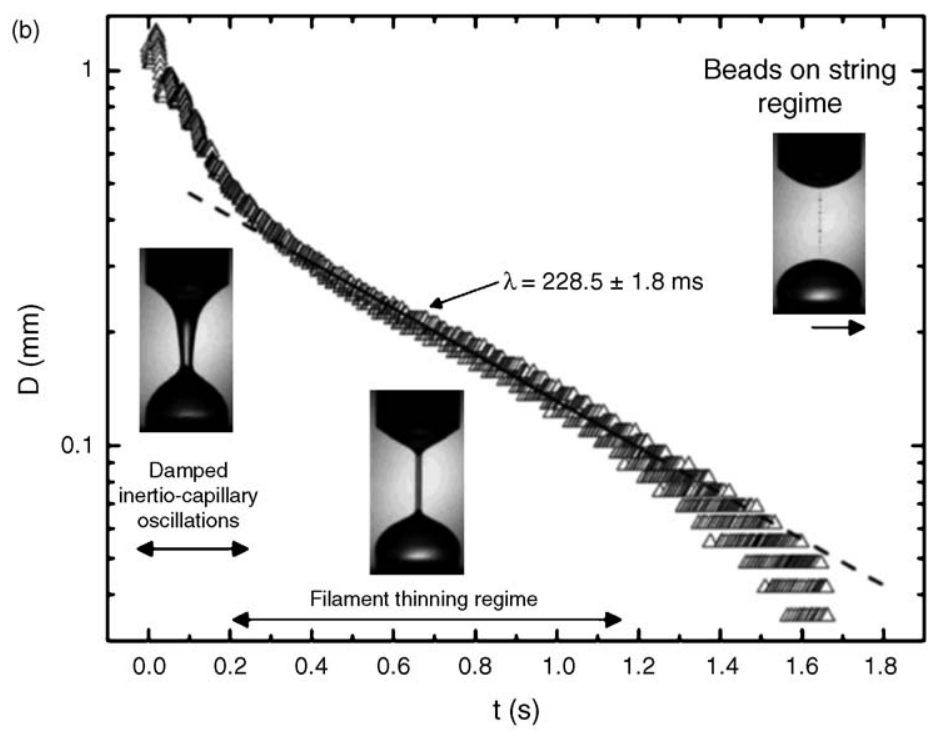

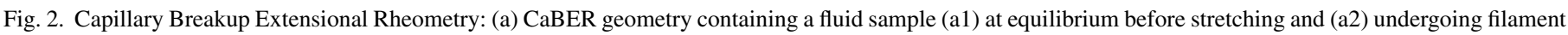

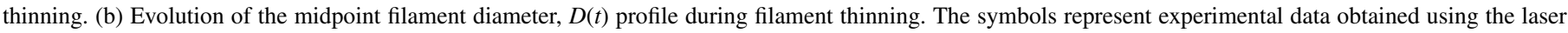
micrometer and the solid line corresponds to the regression using Eq. (1). 


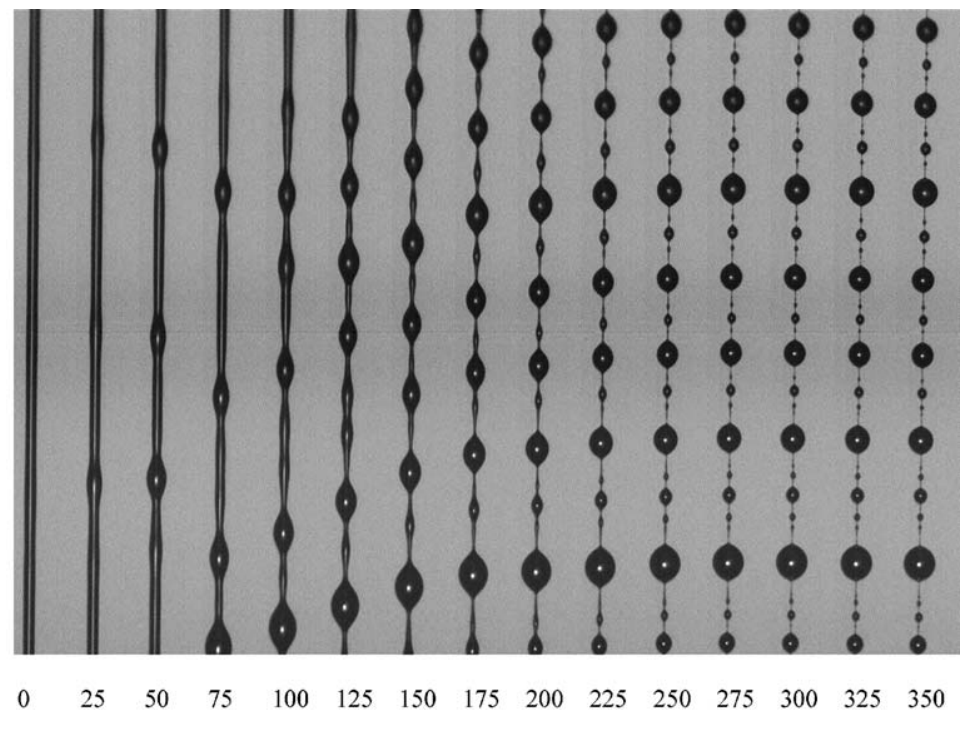

Fig. 3. Sequence of experimental images $(226 \mu \mathrm{m} \times 2317 \mu \mathrm{m})$ of the formation and evolution of beads-on-a-string. Development of the first generation of beadson-a-string occurs at $t=t_{\mathrm{B}} \approx 1.7 \mathrm{~s}$ (first image).

At long times $t \geq t_{\mathrm{B}} \approx 1.7 \mathrm{~s}(t / \lambda \geq 7.5)$ the diameter of the necking thread approaches the characteristic length scale $l \approx 39 \mu \mathrm{m}$ discussed above, at which inertial, capillary and viscous effects are all important. The inset video image in Fig. 2(b) shows that a series of regularly spaced beads spontaneously form on the viscoelastic fluid. The laser micrometer has a minimum measurable diameter of $20 \mu \mathrm{m}$ and the signal/noise ratio becomes increasingly poor at these scales. We therefore utilize the high-resolution digital video images for further analysis.

A sequence of images showing the formation of the beads is presented in Fig. 3. It should be noted that, due to the high magnification necessary to capture the fine-scale structure of the beads-on-a-string, it is not possible to analyze the entire filament. Thus, the snap-shots shown correspond to a section of $2317 \mu \mathrm{m} \times 226 \mu \mathrm{m}$, located near the center of the filament. The repeated or 'iterated' nature of the bead formation process is clear. Following the initial instability and formation of a primary generation of beads the new interconnecting fluid threads become unstable and form a second and third generation of beads. Using image analysis software (ImageJ; NIH), we are able to measure the diameters of the connecting filament at the onset of each bead formation event (denoted henceforth $D_{N}$ for $N=1,2, \ldots$ ), as well as the bead diameters (denoted $B_{n}$ ) for each generation of beads well into the beads-on-a-string regime. The evolution in the bead radius and thread diameter within one period are connected through conservation of mass [12]. The bead diameters for the first three generations are $B_{1} \approx 110 \mu \mathrm{m}$, $B_{2} \approx 75 \mu \mathrm{m}$ and $B_{3} \approx 31 \mu \mathrm{m}$, respectively. A fourth generation is just discernable but hard to quantify as the beads approach the minimum spatial resolution of the image ( 1 pixel $\approx 2.3 \mu \mathrm{m}$ ).

The wavelength of the initial disturbance is very difficult to determine from the small amplitude of the perturbations observed in the first few images, but it can be obtained robustly by measuring the final spacing of the primary generation beads at long times and comparing this with the measured diameter of the filament at the onset of bead formation. For the eight bead structure in Fig. 3 the dimensionless wavelength of the initial disturbance is found to be $\left(L_{\text {beads }} / D_{1}\right)=7.7 \pm 1.4$. Similar values are obtained from other experimental realizations (cf. Fig. 7(a)).

The data obtained from the image analysis software are superimposed onto the laser micrometer measurements in Fig. 4. The formation and growth of each new generation of beads is accompanied by a sharp thinning of the inter-connecting ligaments. This results in a deviation from the exponential decay observed in the earlier elastocapillary regime and appears to result in a close-to-linear decrease in time (see inset in Fig. 4). These characteristics of the iterated stretching sequence are consistent with the predictions of Chang et al. [19]. The theoretical analysis also predicted a recursive relationship $D_{n}=f\left(D_{n-1}\right)$ between the filament diameters at which successive generations form. By assuming that each successive iterated instability developed very rapidly (with no elastocapillary thinning between each generation), Chang et al. obtained the relationship

$\left(D_{n} / d_{0}\right)=\sqrt{2}\left(D_{n-1} / d_{0}\right)^{3 / 2}$

for generations $n \geq 2$; here $d_{0}=2 r_{0}$ is the initial diameter of the filament at the point when the elastocapillary balance is established. This relationship is shown in Fig. 5 for the first four generations formed in six different experimental realizations. Although there does appear to be a recursive relationship between consecutive generations, such that $D_{N} \sim D_{N-1}^{m}$, it is not captured by Eq. (3). However, the data are described to a good approximation by a power law of form:

$\left(D_{N} / D^{*}\right)=\left(D_{N-1} / D^{*}\right)^{m}$

where we have incorporated any numerical front factor present on the right-hand side of Eq. (4) into the definition of $D^{*}$ for clarity. A least-squares fit of Eq. (4) to the experimental data (solid line in Fig. 5) yields an exponent $m=2$ and a characteristic length 


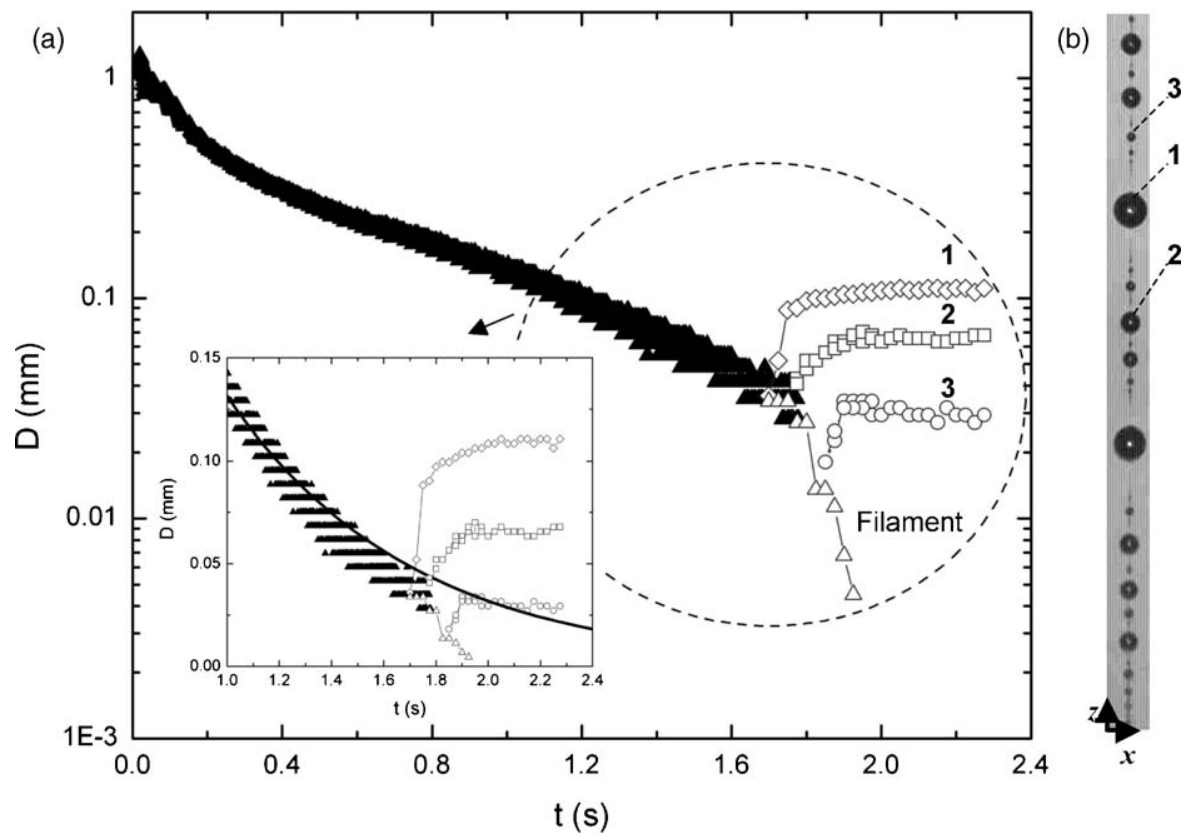

Fig. 4. (a) Evolution of filament and droplet sizes during a CaBER experiment. The filled symbols ( $\mathbf{\Lambda})$ represent filament diameters obtained with the laser micrometer, and the hollow symbols represent measurements obtained using image analysis, corresponding to: $(\triangle)$ filament; $(\diamond)$ first generation bead; $(\square)$ second generation bead and $(\bigcirc)$ third generation bead. The solid line corresponds to the exponential fit of experimental data to Eq. (1). The inset shows the highlighted region plotted on a linear scale. (b) Typical image of beads-on-a-string $(138 \mu \mathrm{m} \times 2317 \mu \mathrm{m})$ used for image analysis captured at $t-t_{\mathrm{B}}=500 \mathrm{~ms}$. The droplets corresponding to those represented in (a) are identified as 1, 2 and 3, for first, second and third generation, respectively.

scale $D^{*} \approx 44 \mu \mathrm{m}$, which is very close to the Eggers length scale $\ell \approx 39 \mu \mathrm{m}$ computed a priori. The deviation between the asymptotic theory and the experimental observations is most likely the result of finite extensibility effects: as the chains approach their maximum elongation, the rate of thinning increases from slow elasto-capillary (exponential) drainage to a linear decay towards a critical breakup time $t_{\mathrm{c}}$. This leads to a more rapid decay in the radius of the thread and thus a higher value of the exponent $m$.

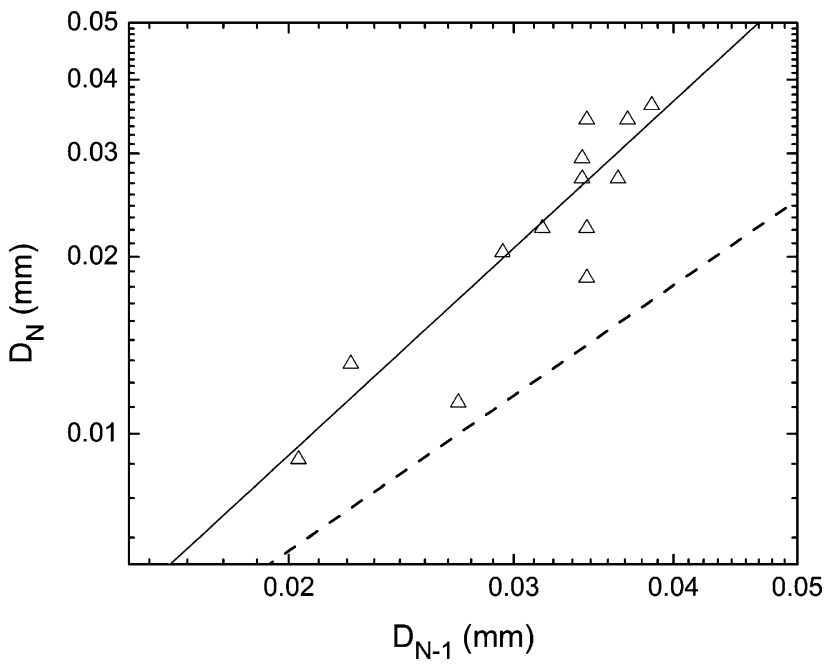

Fig. 5. Recursive relationship for filaments of successive generations obtained from various experiments. The dashed line corresponds to the Chang et al. (1999) prediction given by Eq. (3); and the solid line shows the best power law regression with $D_{1}=44 \mu \mathrm{m}$ and $n=2$ in Eq. (4).
The location of the instability leading to the development of a beads-on-a-string structure is analyzed in more detail in Fig. 6 . The axial profiles of the filament $R(z, t)$ are generated using an edge-detection algorithm and by manually tracking the evolution of a given drop from onset of initial instability until the structure is fully developed. For each time $t_{i}$, the axial position of the primary drop was off-set to center the primary bead (with axial coordinate $\left.z_{\mathrm{B}}(t)\right)$ at the origin of Fig. 6. As the primary bead forms, pinching occurs at the necks on each side of the bead $\left(t-t_{\mathrm{B}}=50 \mathrm{~ms}\right)$. At this point, the filament in the neck region is thinner than in the main thread away from the beads. The filament in the neck gradually recoils $\left(t-t_{\mathrm{B}}=100 \mathrm{~ms}\right)$ and feeds a newly developing bead on each side of the primary one. Meanwhile, the main filament connecting the beads grows thinner and thinner while the main bead is driven by capillarity into an almost spherical shape $\left(t-t_{\mathrm{B}}=500 \mathrm{~ms}\right)$. This process of pinching and recoiling can also be seen for subsequent generations. For the second generation droplets in Fig. 6, for example, pinching is clear at $t-t_{\mathrm{B}}=150$ and $175 \mathrm{~ms}$.

In addition to the iterated stretching, we observe another feature in the dynamics associated with a long time coalescence phase that leads to a 'coarsening' of the beads-on-a-string structure. The smaller, higher-generation beads translate axially along the filament and are 'consumed' by the larger primary droplets. In order to represent the complete spatial and temporal dynamics of this process, from the first stages of droplet formation through coalescence until filament breakage, we follow the approach pioneered by Baumert and Muller [54] and process the stream of digital images to construct a 'space-time' diagram. For each frame $i(=1,2, \ldots, 2048)$ and each axial (vertical) position $z_{\mathrm{j}}(1 \leq j \leq 1024)$, the average gray-scale intensity along the 


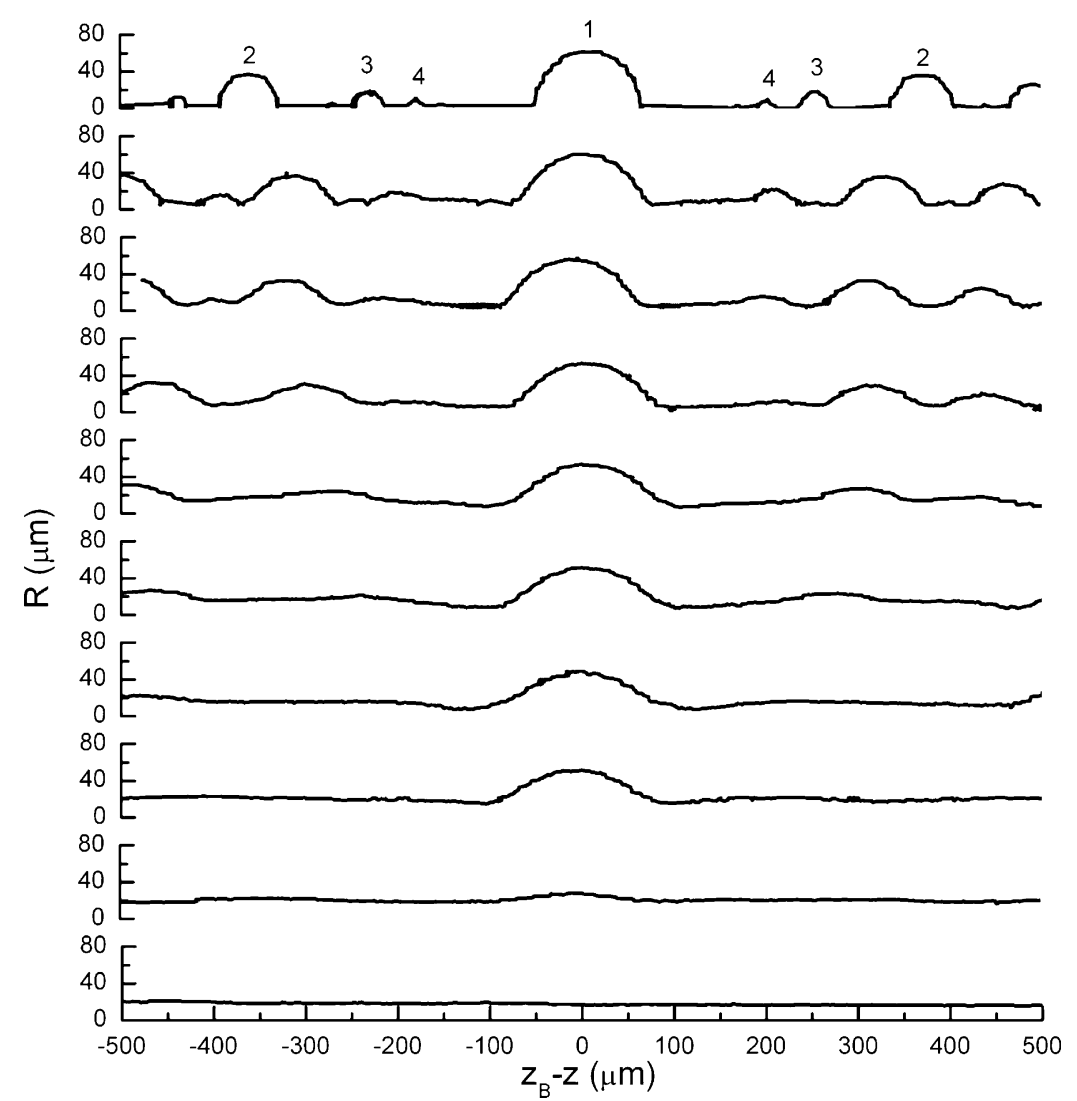

Fig. 6. Progressive evolution of the radius of an initially cylindrical fluid filament produced during a CaBER experiment towards a beads-on-a-string morphology. The relative times of each profile are, $t-t_{\mathrm{B}}: 0,25,50,75,100,125,150,175,200,500 \mathrm{~ms}$, from bottom to top. At each time, the axial position, $z$, has been shifted to show the main bead (with axial coordinate $z_{\mathrm{B}}(t)$ ) at the center of the plot. The numbers on the top graph classify each droplet in terms of its generation.

(horizontal) $x$-axis was calculated. Higher intensities correspond to thicker local slices of the fluid filament (e.g. beads), while low intensities correspond to thin fluid elements (thin filament sections). The average intensities are re-scaled from 0 to 1 , where 0 represents the thinnest filament present and 1 represents the largest bead in the whole process. The resulting column vector of intensities is stored as an entry in an array of size $(i \times j)$ to create a space-time diagram as shown in Fig. 7 that captures the formation of the beads-on-a-string morphology and the associated coarsening. Initially the intensity is homogeneous in $z$, showing the existence of a uniform filament. As the first generation of beads form (at $t_{\mathrm{B}} \approx 2.0 \mathrm{~s}$ ), we see the appearance of bright bands. Higher generation (and hence smaller) beads appear as progressively lower intensity traces. After about $0.4 \mathrm{~s}$, the beads-on-a-string structure is fully established; at this point, there is no visible formation of new beads. However in contrast to the expected rupture event [19] we observe a new regime in which groups of fully formed beads migrate axially along the filament. As a result, coalescence between beads of different generations occurs in accord with recent numerical descriptions of draining and merging of beads [18]. The large relief in elastic tension of the fluid thread following each coalescence event is evidenced by the apparent discontinuities in the bead traces shown in Fig. 7 corresponding to very rapid small-amplitude and affine axial displacements observed along the whole filament (e.g. at $t-t_{\mathrm{B}}=0.45$ and $0.67 \mathrm{~s}$ ). Eventually, when the structure has coarsened to a few large beads, the extensibility limit of the polymer is reached and the filament breaks. This coalescence phase lasts at least $1.4 \mathrm{~s}$ (corresponding to $5 \lambda$ or more); however reproducibility is hard to achieve in this phase because the final rupture of the thin filament is sensitive to the presence of dirt or disruption by air currents.

Finally, we return to the use of elastocapillary thinning and breakup as an extensional rheometer. A balance of elastic and capillary forces in an axially-uniform thread undergoing necking leads to an apparent extensional viscosity that is related to the surface tension $(\sigma)$ and the first derivative of the filament diameter according to $\eta_{\mathrm{app}}=-\sigma /(\mathrm{d} D / \mathrm{d} t)[13,28]$. This is frequently expressed in non-dimensional form as a Trouton ratio, $\operatorname{Tr}=\eta_{\text {app }} / \eta_{0}$. The transient Trouton ratio is shown in Fig. 8 as a function of the total Hencky strain, experienced by a material element $\varepsilon_{\mathrm{H}}=\int_{0}^{t} \dot{\varepsilon}\left(t^{\prime}\right) \mathrm{d} t^{\prime}=-2 \ln \left(D(t) / D_{\mathrm{p}}\right)$ where $D_{\mathrm{p}}$ is the diameter of the endplates (which controls the initial diameter of the relaxed liquid bridge). The open symbols are obtained by numerical differentiation of the experimental data measured for $D(t)$, and become increasingly noisy as the filament diameter decreases and the discrete resolution of the laser micrometer is approached. To overcome this issue, the diameter profile was also fitted to the following empirical expression:

$D=\left(D_{1}+\frac{k_{1}}{t+t_{1}}\right) \exp \left(-\frac{t}{3 \lambda}\right)-V_{2}\left(t-t_{2}\right)$ 


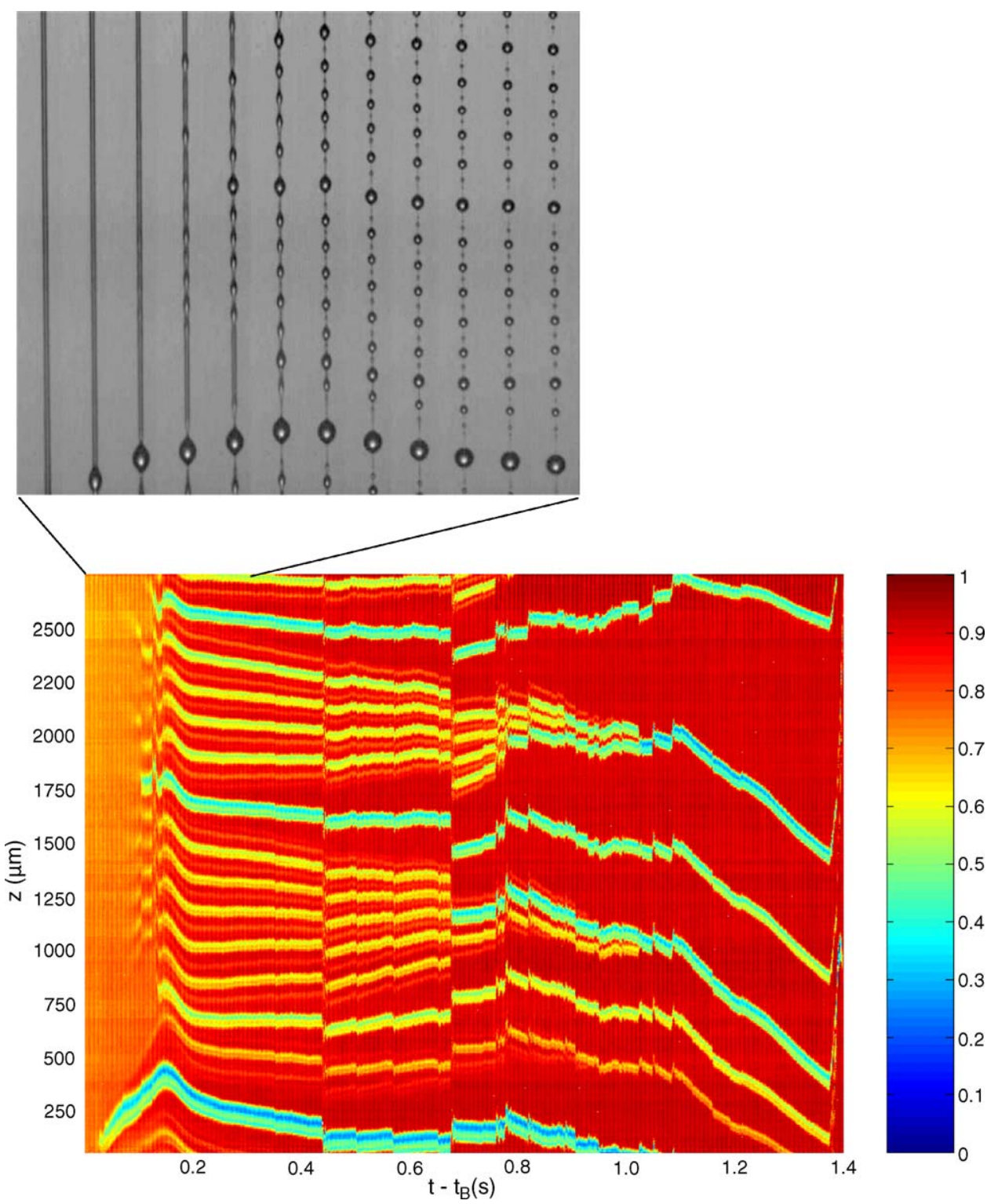

Fig. 7. Construction of a space-time diagram to concisely capture the long time evolution in the filament profile: (a) sequence of experimental images $(263 \mu \mathrm{m} \times 2695 \mu \mathrm{m})$ showing the initial formation and evolution of the beads-on-a-string morphology. Development of the first generation of beads-on-a-string occurs at $t=t_{\mathrm{B}} \approx 2.0 \mathrm{~s}$; (b) Corresponding space-time diagram for this experiment spanning a total elapsed time of $1.4 \mathrm{~s}$. The color of each pixel coordinate $\left\{z_{i}, t_{j}\right\}$ indicates the relative thickness of the filament at that axial position and time; ranging from zero filament thickness (dark red) to the thickest bead (dark blue).

with $D_{1}, t_{1}, k_{1}, V_{2}, t_{2}$ as fitting parameters. This functional form is motivated by the different capillary necking regimes expected theoretically and is able to describe all three phases generically observed in measurements of the midpoint diameter: (i) the rapid initial necking of the filament (for small times $t<t_{1}$ ) after opening as it approaches the elastocapillary balance; (ii) the exponential thinning regimes (for diameters $D \leq D_{1}+k_{1} / t_{1}$ ) as well as (iii) the approach to finite molecular extensibility and onset of the self-similar drainage regime (which is captured by the linear term in Eq. (5), corresponding to an appropriate 'capillary velocity' expected to scale as $V_{2} \sim \sigma / \eta_{\mathrm{E}}$ for a highly stretched polymer solution).

Nonlinear regression to the data yields $D_{1}=0.067 \mathrm{~mm}$, $k_{1}=0.104 \mathrm{~mm} \mathrm{~s}, t_{1}=0.093 \mathrm{~s}, V_{2}=0.1 \mathrm{~mm} \mathrm{~s}^{-1}$ and $t_{2}=1.9 \mathrm{~s}$ as shown by the solid line in the inset to Fig. 8. Analytic differentiation of Eq. (5) and substitution into the expression for the Trouton ratio then results in the solid line shown in the main graph of Fig. 8. The Trouton ratio climbs, initially exponentially, and approaches a steady state value at large strains. The asymptotic limit obtained from Eq. (5) for large strains gives a very large Trouton ratio $\left(\operatorname{Tr}_{\infty}\right)_{\exp } \rightarrow \sigma /\left(\eta_{0} V_{2}\right) \approx 1.3 \times 10^{4}$. This is in good agreement with the range expected for a dilute solution of highly flexible molecules $[29,47]$. For the specific case of FENE dumbbells approaching full stretch in a homogeneous uniaxial extensional flow, we expect $\lim \left(\tau_{p, \mathrm{zz}}-\tau_{p, \mathrm{rr}}\right) \rightarrow \eta_{\mathrm{E}} \dot{\varepsilon}_{0}=2 \eta_{\mathrm{p}} L^{2} \dot{\varepsilon}_{0}$. Substituting this expression into (1) together with the expected linear variation in radius close to breakup $R \rightarrow B\left(t_{\mathrm{c}}-t\right)$ (where $B$ is an unknown constant) and matching the dominant terms, we 


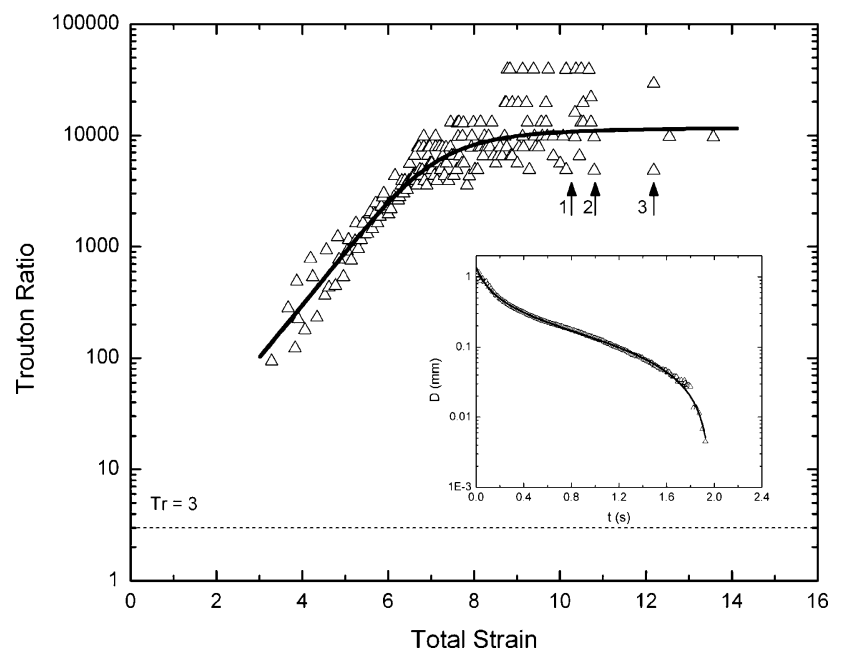

Fig. 8. Apparent dimensionless extensional viscosity obtained from CaBER experiments as a function of the total strain. The symbols are obtained by direct numerical differentiation of the experimental data for the filament diameter, while the solid line is calculated from the analytical derivative of Eq. (5). The labels 1, 2 and 3 indicate where the formation of a first, second and third generation of beads occurs. The inset shows the fit of the experimental data points to Eq. (5).

find that $B=\sigma /\left(4 \eta_{\mathrm{p}} L^{2}\right)=\sigma / 2 \eta_{\mathrm{E}}$ and the asymptotic value of the Trouton ratio predicted by the FENE-P model in a CABER experiment becomes $\operatorname{Tr}_{\infty} \rightarrow 2(1-S) L^{2}$. Substituting for the measured value of $S=\eta_{\mathrm{s}} / \eta_{0}=0.14$ and the theoretical value of $L^{2}=2.4 \times 10^{4}$ we obtain $\left(\operatorname{Tr}_{\infty}\right)_{\text {FENE-P }} \rightarrow 4.1 \times 10^{4}$. This is in reasonable agreement with the experimental value, given the constraints of the theoretical model (which is most suited to a dilute solution of monodisperse flexible chains rather than a semi-dilute solution of polydisperse and possibly aggregating molecules).

The characteristic Hencky strains at which each generation of beads forms are shown by the arrows in Fig. 8 and it is clear that the Trouton ratio is no longer climbing exponentially in this regime. In this fluid, formation of the beads-on-a-string structure occurs concomitantly with the deviation from exponential growth in the elastic stress and the slow approach to full elongation. This is not incorporated in the existing theoretical analysis of the iterated instability leading to bead formation [19].

\section{Conclusions}

In this paper we have shown that analysis of elastocapillary thinning and breakup provides a means of probing the transient extensional response even for very low viscosity - but highly elastic and extensible - polymer solutions (i.e. viscoelastic fluids with $\mathrm{Oh} \ll 1$ but $\mathrm{De} \geq 1$ and $L^{2} \gg 1$ ). At late stages of thinning such fluids are prone to iterated instabilities that result in an array of beads-on-a-string and a subsequent slow axial drainage and consolidation phase. However it is still possible to evaluate the growth in the apparent extensional viscosity and the approach to steady state, provided that a high resolution video-imaging system is used to monitor the continued elasto-capillary thinning in the diameter of the slender elastic threads interconnecting the beads.

Many of the basic features we observe have been described in isolation by existing analyses $[18,19]$; however, the interconnected nature of the iterated instability and coalescence phases has not been analyzed to date. We observe a different recursion relationship (see Fig. 5) from that obtained from asymptotic analysis of the Oldroyd-B equation in the limit of infinite De. This difference in the observed scaling appears to be a result of additional drainage of the interconnecting elastic threads between successive instability events coupled with the finite extensibility of the PEO chains.

The prolonged coalescence phase of the dynamics represented by the space-time diagram (Fig. 6) has also not been described in detail. Li and Fontelos [18] have computed the development of secondary droplets in addition to the slow axial translation (and merging) of the smaller droplets into the larger primary drops. However, these simulations were performed for the infinitely extensible Oldroyd-B model, and thus a finite time breakup event is not admitted. By contrast, our experiments show that for the $2000 \mathrm{ppm}$ PEO solution finite extensibility effects are important in the observed dynamics. Combining the experimental value of the capillary velocity $V_{2}=0.1 \mathrm{~mm} \mathrm{~s}^{-1}$ (fitted to the midpoint diameter data in Fig. 8 over the linearly decreasing regime at late times) and the critical onset diameter $D^{*}=0.044 \mathrm{~mm}$ (fitted to the recursion relationship in Fig. 5) we would expect the entire beads formation and breakup event to last for a time on the order of $\Delta t \approx D^{*} / V_{2}=0.44 \mathrm{~s}$. This is consistent with the period of time over which the recursive bead creation process is observed in Fig. 7(a) and with the expression $t_{2}-t_{\mathrm{B}}$ (where $t_{2}$ is the 'apparent time to breakup' determined by fitting the measured midpoint diameter to Eq. (5)). However, there then follows a prolonged period of coalescence that lasts another 1.5-3 s. Careful measurements of the radii of the interconnecting ligaments in this region is extremely difficult because they approach the resolution of the imaging system. However it is clear that each ligament no longer decays linearly towards a finite time breakup with a slope that is consistent with the steady elongational viscosity of the fluid. The capillary thinning process in the highly stretched polymer solution appears to be arrested by the bead coalescence events. A useful path forward in understanding this process may be to consider the total potential energy of the system. The discrete coalescence events that occur along the fluid column appear to relieve significant amounts of stored elastic energy (as evidenced by the discontinuous jumps in the bead trajectories shown in Fig. 7 which correspond to rapid elastic-like global displacements of the chain of beads). From an energetic point of view, the lowest total energy state is obtained in the largest primary droplets (in which the capillary pressure is lowest and the chains are fully relaxed). In the smaller secondary droplets the capillary pressure (and the associated surface area per volume) is higher, whereas the elastic potential energy of the chains is highest in the thin stretched ligaments. It thus appears that this final stage of the dynamics is cooperative as the entire structure of multiple generations of different sized beads on a string relaxes towards a final global minimum energy configuration. It is to be hoped that these final stages of the drainage and 
breakup of polymer threads will be described by future analytic and numerical studies.

\section{Acknowledgements}

The authors would like to thank Prof. E.S.G. Shaqfeh's research group for providing the polymer solution used in this work. M.S.N. Oliveira wishes to acknowledge Fundação para Ciência e Tecnologia (Portugal) for financial support. The high speed video imaging facilities utilized in this work were acquired under an NSF-MRI grant CTS-0116486.

\section{References}

[1] S. Middleman, Stability of a viscoelastic jet, Chem. Eng. Sci. 20 (1965) 1037-1040.

[2] M. Goldin, H. Yerushalmi, R. Pfeffer, R. Shinnar, Breakup of a laminar capillary jet of a viscoelastic fluid, J. Fluid Mech. 38 (4) (1969) 689-711.

[3] Y. Amarouchene, D. Bonn, J. Meunier, H. Kellay, Inhibition of the finite time singularity during droplet fission of a polymeric fluid, Phys. Rev. Lett. 86 (16) (2001) 3558-3562.

[4] M. Renardy, A numerical study of the asymptotic evolution and breakup of Newtonian and viscoelastic jets, J. Non-Newt. Fluid Mech. 59 (1995) 267-282.

[5] J.W. Hoyt, J.J. Taylor, R.L. Altman, Drag reduction-jet breakup correlation with kerosene-based additive, J. Rheol. 24 (1980) 685-705.

[6] R.P. Mun, B.W. Young, D.V. Boger, Atomisation of dilute polymer solutions in agricultural spray nozzles, J. Non-Newt. Fluid Mech. 83 (1-2) (1999) 163-178

[7] V. Romagnoli, P. Felton, R.K. Prud'homme, Effect of additives on secondary breakup of droplets in high-speed airflows, in: Proceedings of the Eighth International Conference on Liquid Atomization and Spray Systems, Pasadena, CA, 2000.

[8] R.H. Fernando, L.-L. Xing, J.E. Glass, Rheology parameters controlling spray atomization and roll-misting behavior of waterborne coatings, Prog. Org. Coatings 42 (2001) 244-248.

[9] H. Fong, I. Chun, D.H. Reneker, Beaded nanofibers formed during electrospinning, Polymer 40 (16) (1999) 4585-4592.

[10] H.J. Shore, G. Harrison, The effect of added polymer on the formation of drops ejected from a nozzle, Phys. Fluids 17 (2005) 033104.

[11] A.L. Yarin, Free Liquid Jets and Films: Hydrodynamics and Rheology. Interaction of Mechanics and Mathematics Series Longman, Wiley, New York, 1993.

[12] M.C. Sostarecz, A. Belmonte, Beads-on-String phenomena in wormlike micellar fluids, Phys. Fluids 16 (9) (2004) L67-L71.

[13] P. Schümmer, K.H. Tebel, A new elongational rheometer for polymer solutions, J. Non-Newt. Fluid Mech. 12 (1983) 331-347.

[14] Y. Christanti, L. Walker, Surface tension driven jet break up of strainhardening polymer solutions, J. Non-Newt. Fluid Mech. 100 (1-3) (2001) 9-26.

[15] Y.M. Christanti, L. Walker, Effect of fluid relaxation time on jet breakup due to a forced disturbance of polymer solutions, J. Rheol. 46 (3) (2002) 733-748.

[16] M. Renardy, Some comments on the surface-tension driven breakup (or the lack of it) of viscoelastic jets, J. Non-Newt. Fluid Mech. 51 (1994) 97-107.

[17] D.W. Bousfield, R. Keunings, G. Marrucci, M.M. Denn, Nonlinear analysis of the surface-tension driven breakup of viscoelastic fluid filaments, J. Non-Newt. Fluid Mech. 21 (1986) 79-97.

[18] J. Li, M.A. Fontelos, Drop dynamics on the beads-on-string structure for viscoelastic jets: a numerical study, Phys. Fluids 15 (4) (2003) 922-937.

[19] H.C. Chang, E.A. Demekhin, E. Kalaidin, Iterated stretching of viscoelastic jets, Phys. Fluids 11 (7) (1999) 1717-1737.
[20] G.H. McKinley, Visco-elasto-capillary thinning and breakup of complex fluids, in: D.M. Binding, K. Walters (Eds.), Rheology Reviews, Aberystwyth, 2005, pp. 1-48.

[21] M.P. Brenner, X.D. Shi, S.R. Nagel, Iterated instabilities during droplet fission, Phys. Rev. Lett. 73 (25) (1994) 3391-3394.

[22] X.D. Shi, M.P. Brenner, S.R. Nagel, A cascade of structure in a drop falling from a faucet, Science 265 (5169) (1994) 219-222.

[23] D'Arcy Thompson, On growth and form, in: J.T. Bonner (abridged Ed.), CUP, Cambridge, 1961.

[24] M.S.N. Oliveira, G.H. McKinley, Iterated stretching and multiple beadson-a-string phenomena in dilute solutions of highly-extensible flexible polymers, Phys. Fluids (Lett.) 17 (7) (2005) 071704.

[25] A.V. Bazilevsky, V.M. Entov, A.N. Rozhkov, Liquid filament microrheometer and some of its applications, in: D.R. Oliver (Ed.), Proceedings of the Third European Rheology Conference, Elsevier Applied Science, 1990, pp. 41-43.

[26] M. Stelter, G. Brenn, A.L. Yarin, R.P. Singh, F. Durst, Validation and application of a novel elongational device for polymer solutions, J. Rheol. 44 (3) (2000) 595-616.

[27] M. Stelter, G. Brenn, A.L. Yarin, R.P. Singh, F. Durst, Investigation of the elongational behavior of polymer solutions by means of an elongational rheometer, J. Rheol. 46 (2) (2002) 507-527.

[28] S.L. Anna, G.H. McKinley, Elasto-capillary thinning and breakup of model elastic liquids, J. Rheol. 45 (1) (2001) 115-138.

[29] A. Lindner, J. Vermant, D. Bonn, How to obtain the elongational viscosity of dilute polymer solutions, Physica A 319 (2003) 125-133.

[30] J.J. Cooper-White, J.E. Fagan, V. Tirtaatmadja, D.R. Lester, D.V. Boger, Drop formation dynamics of constant low-viscosity, elastic fluids, J. Non-Newt. Fluid Mech. 106 (1) (2002) 29-59.

[31] L.E. Rodd, T.P. Scott, J.J. Cooper-White, G.H. McKinley, Capillary breakup rheometry of low-viscosity elastic fluids, Appl. Rheol. 15 (1) (2005) 12-27.

[32] R.G. Larson, The Structure and Rheology of Complex Fluids, Oxford University Press, New York, 1999.

[33] E.B. Chen, A.J. Morales, C.-C. Chen, A.A. Donatelli, W.W. Bannister, B.T. Cummings, Fluorescein and poly(ethylene oxide) hose stream additives for improved firefighting effectiveness, Fire Tech. 34 (4) (1998) 291-307.

[34] V. Tirtaatmadja, G.H. McKinley, J.J. Cooper-White, Drop formation and breakup of low viscosity elastic fluids: effects of concentration and molecular weight, Phys. Fluids (2006), in press.

[35] M.J. Solomon, Personal Communication, 2005.

[36] F.E. Bailey, J.L. Kucera, L.G. Imhof, Molecular weight relations of poly(ethylene oxide), J. Polym. Sci. 32 (1958) 517.

[37] H.P. Sdougos, S.R. Bussolari, C.F. Dewey, Secondary flow and turbulence in a cone-and-plate device, J. Fluid Mech. 138 (1984) 379-404.

[38] G.H. McKinley, A. Öztekin, J.A. Byars, R.A. Brown, Self-similar spiral instabilities in elastic flows between a cone and a plate, J. Fluid Mech. 285 (1995) 123-164.

[39] D.O. Olagunju, Hopf bifurcation in creeping cone-and-plate flow of a viscoelastic fluid, Z.A.M.P. 48 (3) (1997) 361-369.

[40] G.H. McKinley, P. Pakdel, A. Öztekin, Geometric and rheological scaling of purely elastic flow instabilities, J. Non-Newt. Fluid Mech. 67 (1996) 19-48.

[41] K.C. Tam, C. Tiu, Steady and dynamic shear properties of aqueous polymer solutions, J. Rheol. 33 (2) (1989) 257-280.

[42] K.C. Tam, C. Tiu, Improved correlation for shear-rate dependent viscosity of polyelectrolyte solutions, J. Non-Newt. Fluid Mech. 46 (1993) 275-288.

[43] V.N. Kalashnikov, Shear-rate dependent viscosity of dilute polymer solutions, J. Rheol. 38 (5) (1993) 1385-1403.

[44] A.V. Bazilevskii, V.M. Entov, A.N. Rozhkov, Failure of an Oldroyd liquid bridge as a method for testing the rheological properties of polymer solutions, Polym. Sci. Ser. A 43 (7) (2001) 1161-1172 (translated from Vysokomolekulyarnye Soedineniya Ser. A, pp. 474-482).

[45] J. Eggers, Nonlinear dynamics and breakup of free-surface flows, Rev. Mod. Phys. 69 (3) (1997) 865-929. 
[46] G.H. McKinley, A. Tripathi, How to extract the Newtonian viscosity from capillary breakup measurements in a filament rheometer, J. Rheol. 44 (3) (2000) 653-671.

[47] R.B. Bird, R.C. Armstrong, O. Hassager, Dynamics of Polymeric Liquids, Fluid Mechanics, vol. 1, 2nd ed., Wiley Interscience, New York, 1987.

[48] V.M. Entov, E.J. Hinch, Effect of a spectrum of relaxation times on the capillary thinning of a filament of elastic liquid, J. Non-Newt. Fluid Mech. 72 (1) (1997) 31-54.

[49] M. Renardy, Self-similar breakup of non-Newtonian fluid jets, in: D.M. Binding, K. Walters (Eds.), Rheology Reviews, The British Society of Rheology, 2004, pp. 171-196.
[50] V.M. Entov, E.J. Hinch, Effect of a spectrum of relaxation times on the capillary thinning of a filament of elastic liquid, J. Non-Newt. Fluid Mech. 72 (1) (1997) 31-53.

[51] M. Renardy, Similarity solutions for jet breakup for various models of viscoelastic fluids, J. Non-Newt. Fluid Mech. 104 (1) (2002) 65-74.

[52] M.A. Fontelos, J. Li, On the evolution and rupture of filaments in Giesekus and FENE models, J. Non-Newt. Fluid Mech. 118 (1) (2004) $1-16$.

[53] S. Chandrasekhar, Hydrodynamic and Hydromagnetic Stability, Dover, New York, 1961

[54] B.M. Baumert, S.J. Muller, Flow visualization of the elastic TaylorCouette instability in Boger fluids, Rheol. Acta 34 (2) (1995) 147-159. 